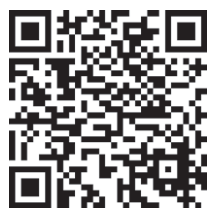

Palabras clave: Otoplastia, orejas prominentes, orejas aladas, simulación quirúrgica, entrenamiento quirúrgico, bajo costo.

Keywords: Otoplasty, prominent ears, winged ears, surgical simulation, surgical training, low cost.

\footnotetext{
* Residente de
} Cirugía Plástica y Reconstructiva, División de Cirugía de la Pontificia Universidad Católica de Chile. \$Cirujano Plástico y Reconstructivo del Hospital Sótero del Río. $\S$ Médico Cirujano de la Pontificia Universidad Católica de Chile.

॥ Cirujano Plástico y Reconstructivo. Profesor asistente adjunto. División de Cirugía de la Pontificia Universidad Católica de Chile.

๑ Cirujano Plástico y Reconstructivo. Profesor asistente. División de Cirugía de la Pontificia Universidad Católica de Chile.

Recibido: 28/01/2020 Aceptado: 05/03/2020

doi: $10.35366 / 92934$

\title{
Diseño y validación de un novedoso modelo de simulación para otoplastia a bajo costo
}

\author{
Development and validation of a novel low cost otoplasty simulation model
}

A Navia,* A López," S Canahuate, ${ }^{\S}$ S Searle,,$\|$ B Dagninoף

\section{RESUMEN}

\section{ABSTRACT}

Introducción: La otoplastia es un procedimiento que se usa para corregir las orejas prominentes. Para aprenderlo, la simulación es un método que ha revolucionado la educación médico-quirúrgica. Sin embargo, hasta donde sabemos, no existe un modelo de otoplastia adecuadamente diseñado y validado. Objetivos: Desarrollar y validar un modelo de otoplastia a bajo costo. Material y métodos: Diseñamos un modelo sintético de oreja prominente. Para ello, mediante un método Delphi, creamos una escala específica para otoplastia (EVOPUC) y un cuestionario de antropometría (CLA). Evaluamos a cinco expertos (cirujanos plásticos) y cinco principiantes (estudiantes). Cada participante realizó un procedimiento que fue grabado y evaluado por tres expertos cegados utilizando una escala general (OSATS), la EVOPUC, el CLA y el tiempo operatorio. Los principiantes realizaron cuatro sesiones de entrenamiento con expertos y fueron reevaluados bajo la misma metodología. Los expertos completaron una encuesta sobre validez de apariencia y contenido. Resultados: El costo de cada modelo fue de cinco dólares. Tras el entrenamiento, los novatos mejoraron significativamente su desempeño $(\mathrm{p}<$ 0.05), el cual fue comparable con el de los expertos. $100 \%$ de los expertos calificó el modelo como útil, y lo consideró una herramienta clave para el entrenamiento de residentes. Conclusiones: Desarrollamos un modelo de otoplastia a bajo costo, el cual logra una adquisición significativa de habilidades en el grupo objetivo con resultados similares a los de cirujanos plásticos experimentados.
Introduction: Otoplasty is a procedure to correct prominent ears. Simulation has acquired great relevance in medical and surgical education. To our knowledge, no otoplasty simulation model has been properly designed nor validated. Objectives: Develop and validate a low cost otoplasty simulation model. Material and methods: We designed a prominent ear synthetic model. A Delphi method was used to create a specific scale for otoplasty (EVOPUC) and an Anthropometry Likert Questionnaire (CLA). Ten participants were recruited, 5 experts (plastic surgeons) and 5 novices (students). Each participant was recorded performing an otoplasty on the model and then assessed by 3 blind experts using a general scale (OSATS), EVOPUC, CLA and the operative time. Novices carried out four guided training sessions with experts. Then they were reassessed using the same methodology. Additionally, experts completed a survey for face and content validation. Results: The cost for each model was 5 dollars. After training, novices significantly improved their performance $(p<0.05)$, comparable to experts. $100 \%$ of the experts rated the model as very useful, being considered as a tool for residents training. Conclusions: We developed a lowcost otoplasty simulation model, achieving a significant acquisition of skills in the targeted group, with results similar to experienced plastic surgeons.

\section{INTRODUCCIÓN}

$\mathrm{L}$ as orejas prominentes o aladas son un tipo de deformación auricular que suele ocurrir hasta en $5 \%$ de la población. ${ }^{1,2}$ Este tipo de deformación corresponde a una displasia grado I o una deformidad tipo $V$ según la clasificación de Weerda o de Tanzer. ${ }^{3,4}$ Tal fenómeno se debe principalmente a uno de tres factores: ausencia de definición de pliegue del antihélix (PAH), hiperplasia de la concha (con aumento del ángulo aurículo-cefálico [AAC], el cual, según los criterios de Wodak y Farkas, es normal entre 20 y $30^{\circ}$ ) o lóbulo prominente (Figura 1).5-7 Para corregirlo, la otoplastia es la cirugía de elección para estas deformidades, y su técnica descrita ha sido múltiple; ${ }^{8-12}$ si bien todas siguen el mismo principio (corregir estas tres deformidades), el plegamiento del antihélix de Mustardé y las suturas de fijación concha-mastoidea de Furnas son las técnicas más populares. ${ }^{13,14}$

El entrenamiento quirúrgico en modelos simulados es ampliamente validado por la literatura para la adquisición de habilidades que permitan 
Abreviaturas:

$\mathrm{AAC}=$ Ángulo

aurículo-cefálico.

CLA = Cuestionario

Likert de Antropometría.

EVOPUC $=$ Escala

de Valoración de

Otoplastia de la

Pontificia Universidad

Católica de Chile.

OSATS $=$ Objective

Structured Assessment

of Technical Skill.

$\mathrm{PAH}=$ Pliegue

del antihélix. tratar las orejas prominentes, convirtiéndolo en una herramienta clave en la educación médica de los residentes. Pese a ello, se han publicado pocas investigaciones que describan su experiencia en la simulación de la otoplastia. ${ }^{15,16}$ Esto se debe a que, por un lado, los modelos animales poseen una desventaja anatómica obvia, así como disponibilidad y almacenamiento más complejos. Con respecto a modelos sintéticos, la literatura disponible muestra una debilidad común: todos presentan una sola capa que no emula la piel y el cartílago por separado, además de que no cuentan con una adecuada validación. ${ }^{17-23}$ Por otro lado, el uso de cadáveres humanos está limitado por el costo y la fortuna de encontrar alguno que presente las deformidades características. ${ }^{24,25}$

Considerando que nuestro grupo ya cuenta con experiencia previa en el desarrollo y validación de programas de entrenamiento ${ }^{26}$ y teniendo en cuenta lo dicho previamente, el objetivo de este estudio fue desarrollar un nuevo modelo de simulación para otoplastia que fuese de bajo costo y a su vez, evaluar su validez de apariencia, contenido y constructo.

\section{MATERIAL Y MÉTODOS}

\section{Diseño y desarrollo del modelo de otoplastia}

Con base en referencias anatómicas, se esculpió en arcilla una oreja izquierda con ausencia del PAH y con un AAC de 65 grados. Luego, se confeccionó un molde negativo con silicona industrial. Se utilizó una mezcla de colorantes y siliconas flexibles curadas a base de platino (Smooth-On Inc., Pennsylvania, EUA) para construir el cartílago y la capa de piel. Después, se agregó una malla de nailon como marco flexible interno para darle más resistencia al cartílago al momento de pasar la aguja de sutura. Por último, el modelo se montó sobre una base de poliestireno con una cubierta de cuero sintético blanco para que emulara la mastoides y el periostio.

\section{Desarrollo de una escala y cuestionario específico de evaluación para otoplastia}

Se utilizó un método Delphi para lograr un consenso entre expertos, para que de esta forma se determinaran los pasos cruciales del PAH (Mustardé) y las técnicas de sutura para la fijación concha-mastoidea (Furnas). Así se creó la Escala de Valoración para Otoplastia de la Pontificia Universidad Católica (EVOPUC). Además, se desarrolló un Cuestionario Likert de Antropometría (CLA) específico para evaluar el grado de corrección antropométrica postoperatoria del PAH y AAC.

Dado que no se encontró una definición en la literatura disponible de "experto" en otoplastia, consideramos que es "alguien que ha realizado el procedimiento en múltiples oportunidades, además de que conoce sus indicaciones, diferentes técnicas, complicaciones y es capaz de enseñarlo a otros". De esta manera, todos los cirujanos plásticos que participaron en este proyecto $(\mathrm{n}=$ 5) cumplieron con este criterio cualitativo.

Figura 1:

Anatomía normal del pabellón auriculary deformidades características de las orejas
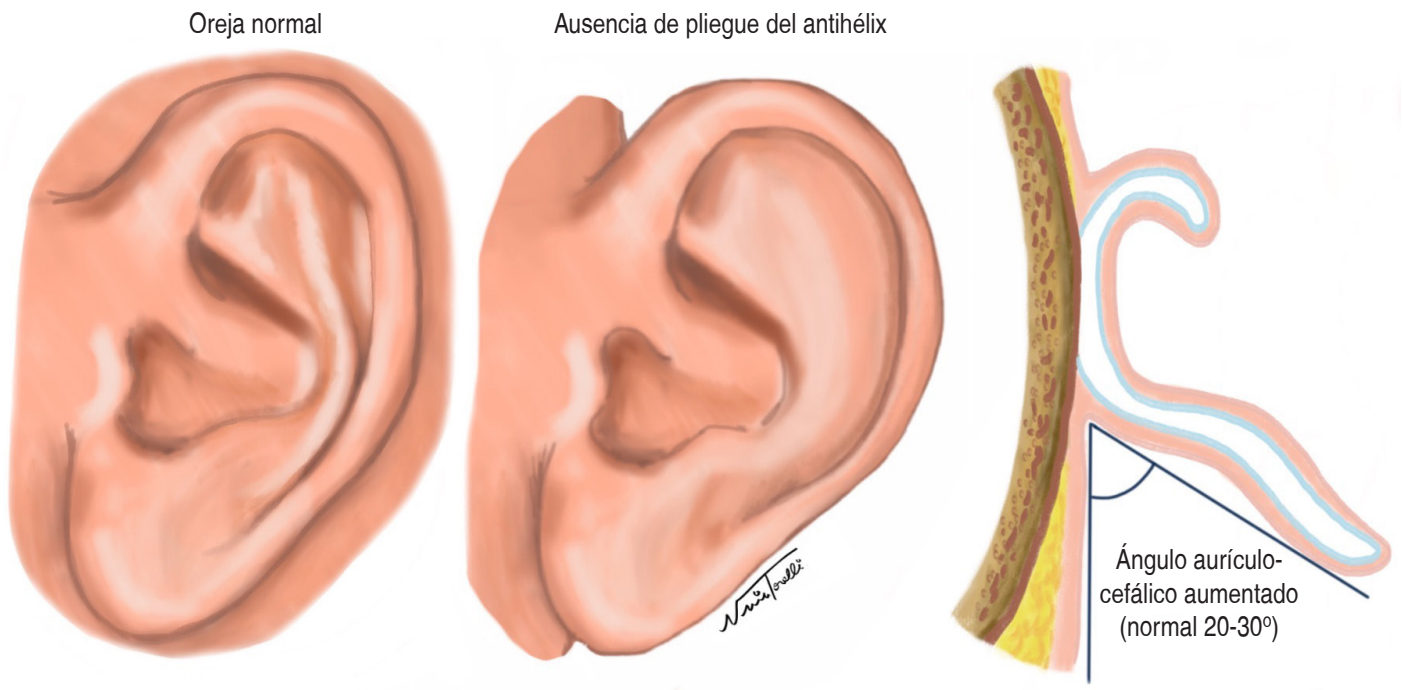
prominentes. 


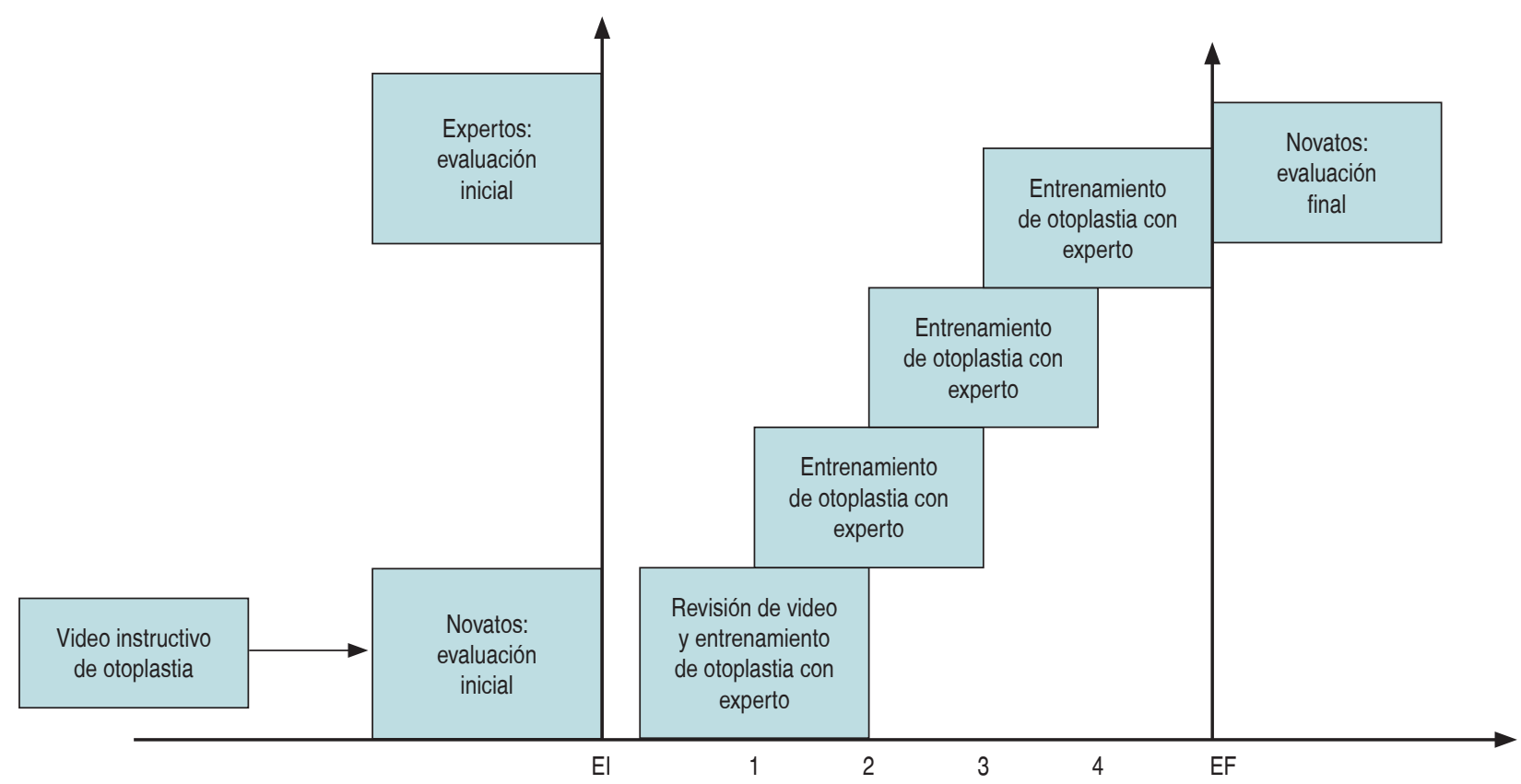

Figura 2: Diseño del programa de entrenamiento en el modelo de otoplastia. EI = evaluación inicial; EF = evaluación final.

\section{Validación del modelo de otoplastia}

\section{Evaluación inicial}

Se reclutaron en total 10 participantes y de éstos, se definieron dos grupos: expertos (cirujanos plásticos $[n=5])$ y principiantes (estudiantes de medicina de quinto año $[n=5])$. Como el grupo de principiantes no tenía conocimiento previo del procedimiento, se desarrolló un video instructivo básico enfocado en la anatomía del pabellón auricular, las deformidades asociadas a las orejas prominentes y una demostración de la técnica quirúrgica por un experto en el modelo de otoplastia (Video suplementario 1).

https://drive.google.com/drive/folders/1 L4ZW X6d7f4FHTuc6nwsNw4chBbGWo2iJ

Para el entrenamiento, se seleccionaron las técnicas del plegado de antihélix de Mustardé y las suturas de fijación concha-mastoideas de Furnas. Antes de realizar la prueba de evaluación inicial en el modelo de otoplastia, los novatos tuvieron la oportunidad de hacer preguntas a un supervisor experto mientras veían el video instructivo. A todos los participantes se les entregó el mismo instrumental quirúrgico. Todos los procedimientos se grabaron en video con una cámara frontal GoPro Hero 3 (GoPro Inc., California, EUA). El primer autor (AN) participó como asistente quirúrgico en todos los procedimientos. La ayuda y grado de participación del asistente quirúrgico debía solicitarse de manera explícita por quien realizaba el procedimiento.

Los videos fueron editados mínimamente, sólo se eliminó el audio, los cuales fueron codificados y ordenados al azar por una persona externa. Para evaluar los resultados antropométricos de los modelos se tomaron fotografías estandarizadas desde el superior y el lateral a una distancia de $10 \mathrm{~cm}$ con un iPhone 6S (Apple Inc., California, EUA). El AAC fue medido digitalmente con Adobe Photoshop CS6 (Adobe Inc., California, EUA) y corroborado por dos evaluadores, logrando un acuerdo sobre todas las mediciones. Todos los videos y fotografías fueron evaluados por tres expertos cegados. Utilizamos la escala Objective Structured Assessment of Technical Skill (OSATS) ${ }^{27}$ para la evaluación de la destreza quirúrgica general, la escala específica EVOPUC, el cuestionario CLA y además se midió el tiempo total en completar el procedimiento.

\section{Sesiones de entrenamiento para principiantes}

Se diseñó un plan de entrenamiento completo según se indica en la Figura 2. Posteriormente, cada miembro del grupo de principiantes revisó su video inicial junto con un experto. Luego de revisar el video, se realizaron cuatro sesiones 
Tabla 1: Cuestionario Likert de antropometría utilizado por evaluadores.

\begin{tabular}{|c|c|c|c|c|c|}
\hline \multicolumn{6}{|c|}{ Cuestionario Likert de Antropometría en Otoplastia } \\
\hline Antropometría & $1=$ muy mal & $2=\mathrm{mal}$ & $3=$ promedio & $4=$ bien & 5 = muy bien \\
\hline
\end{tabular}

Tabla 2: La escala de valoración para otoplastia de la Pontificia Universidad Católica.

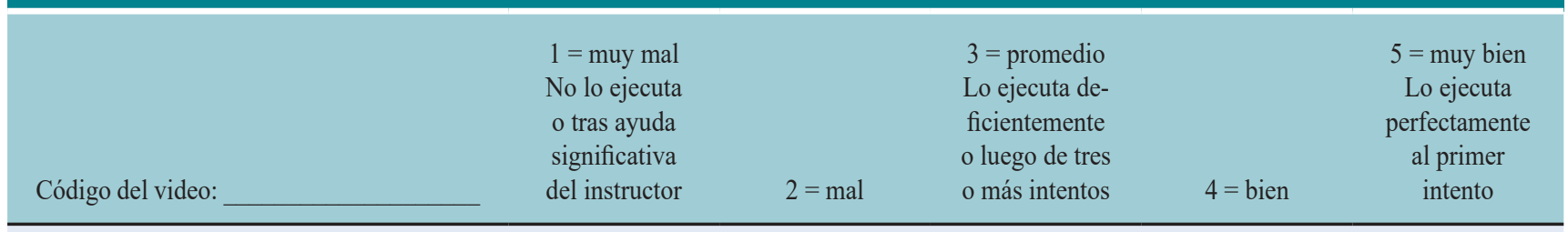

\section{Marcación de la piel}

A) Diseña y realiza incisión elíptica o transversa de la piel

2. Resección de la piel

A) Disección de la piel del cartílago (con tijera o bisturí)

3. Disección auricular posterior

A) Eversión adecuada de los bordes

B) Disección del cartílago del plano cutáneo

C) Extensión adecuada del plano de disección

D) Correcta exposición del periostio de la mastoides

4. Creación del pliegue anterior del antihélix

A) Plegado manual (con pinzas o agujas) de nuevo antihélix

B) Marcación en cartílago de puntos de sutura

5. Sutura del nuevo antihélix

A) Sutura del nuevo antihélix según técnica de Mustardé

B) Verificación de indemnidad de la piel por cara anterior

6. Posicionamiento auricular-fijación concho mastoidal

A) Fijación concho-mastoidal según técnica de Furnas

B) Verificación de indemnidad de la piel por cara anterior

C) Fijación de nudos con adecuada tensión y corrección del ángulo aurículo-cefálico 
de entrenamiento individuales para cada uno, repitiendo el mismo procedimiento, pero con la tutorización de los expertos.

\section{Evaluación final}

Una vez que los principiantes finalizaron las sesiones de entrenamiento, fueron reevaluados utilizando la misma metodología descrita. Los resultados se compararon con sus puntajes previos y con los puntajes de los expertos. Para evaluar la validez de apariencia y contenido, se aplicaron encuestas Likert de cinco puntos a los expertos a través de formularios de Google (Google Inc., California, EUA).

\section{Análisis estadístico}

Los resultados se analizaron con la versión 22.0 del Paquete Estadístico de Ciencias Sociales (SPSS Inc., Illinois, EUA). Como las variables introducidas eran no-paramétricas, se utilizó el test de Wilcoxon para comparar el grupo de novatos antes y después del entrenamiento, y el test de Mann-Whitney para comparar los puntajes entre expertos y novatos después del entrenamiento; un valor de $p<0.05$ se consideró estadísticamente significativo.

El coeficiente de correlación intraclase $(\mathrm{CCl})$ se utilizó para evaluar el acuerdo interobservador de los tres expertos evaluadores. Éste se calculó utilizando un modelo aleatorio de dos vías, que se caracteriza por un acuerdo absoluto para cuantificar la concordancia de los evaluadores con base en los promedios de las calificaciones. Los valores del coeficiente de correlación intraclase se expresaron con un intervalo de confianza (IC) de 95\%.

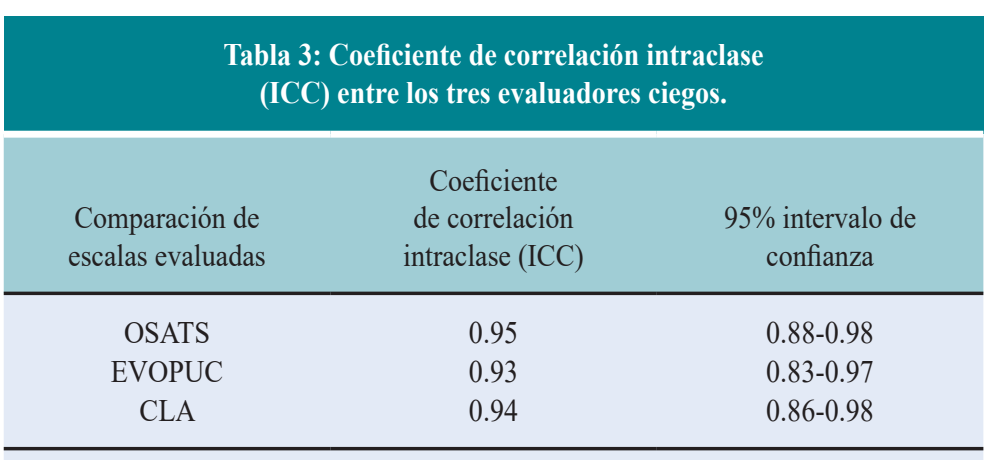

OSATS = Objective Structured Assessment of Technical Skill; EVOPUC = Escala de Valoración de Otoplastia de la Pontificia Universidad Católica de Chile; CLA = Cuestionario Likert de Antropometría.

\section{RESULTADOS}

\section{Diseño y desarrollo del modelo de otoplastia}

Para el modelo se construyó una oreja izquierda de silicona flexible con dos capas, piel y cartílago, y un AAC de 65 grados sin PAH. Cada modelo fue hecho a mano cuyo tiempo de trabajo fue de 30 minutos por modelo (sin considerar los tiempos de curado de la silicona). El costo total de cada modelo fue de aproximadamente cinco dólares americanos.

\section{Desarrollo de una escala y cuestionario de evaluación específica para otoplastia}

Tanto EVOPUC como CLA consisten en escalas Likert de cinco puntos. El cierre de la piel y curación postoperatoria no fueron considerados en la evaluación del procedimiento (Tablas 1 y 2).

\section{Validación del modelo de otoplastia}

\section{Evaluación inicial}

El ICC para determinar el grado de acuerdo entre los tres evaluadores ciegos respecto a las escalas OSATS, EVOPUC y CLA fue de 0.94 (Tabla 3). ${ }^{28}$ La interpretación de CCI se realizó según lo propuesto por Fleiss y Cicchetti, considerando un valor $\geq 0.75$ como un excelente acuerdo entre evaluadores más allá del azar. ${ }^{29,30}$ Los resultados de las evaluaciones según OSATS, EVOPUC, CLA, los tiempos operatorios totales y su análisis estadístico se exponen como mediana (cuartil 1 a 3) en la Tabla 4.

\section{Sesiones de entrenamiento para principiantes}

Todos los novatos completaron el programa de entrenamiento (100\% de seguimiento).

\section{Evaluación final}

Tres expertos analizaron de manera ciega un total de 15 videos, incluida la evaluación inicial de expertos y de principiantes, además de la evaluación final de los principiantes posterior al entrenamiento. Cada principiante realizó seis otoplastias durante todo el estudio.

La comparación entre los puntajes obtenidos por los principiantes en la evaluación inicial, la 
Tabla 4: Resultados de rendimiento para principiantes y expertos en el modelo de otoplastia.

\begin{tabular}{|c|c|c|c|c|c|}
\hline & $\begin{array}{c}\text { Novatos EI } \\
(\mathrm{Q} 1-\mathrm{Q} 3)\end{array}$ & $\begin{array}{c}\text { Novatos EF } \\
\text { (Q1-Q3) }\end{array}$ & $\begin{array}{c}\text { Expertos }^{\mathrm{C}} \\
(\mathrm{Q} 1-\mathrm{Q} 3)\end{array}$ & p A-B* & $\mathrm{p} \mathrm{B}-\mathrm{C} \dagger$ \\
\hline Número & 5 & 5 & 5 & & \\
\hline OSATS (7-35) & $11(9.5-12.5)$ & $25(22-27)$ & $34(32.4-34)$ & 0.043 & 0.008 \\
\hline $\begin{array}{l}\text { EVOPUC } \\
(13-65)\end{array}$ & $39(35.5-40.5)$ & $60(57.5-61.5)$ & $63(60.5-64)$ & 0.042 & 0.095 \\
\hline $\begin{array}{l}\text { Tiempo } \\
\text { (minutos) }\end{array}$ & 43 & 30 & 23 & 0.043 & 0.095 \\
\hline $\begin{array}{l}\text { Antropometría } \\
\text { (CLA) (2-10) }\end{array}$ & $4(3-6)$ & $9(8.5-9)$ & $10(9-10)$ & 0.038 & 0.095 \\
\hline
\end{tabular}

$\mathrm{p}<0.05$ considerado estadísticamente significativo.

* Obtenido al comparar columnas A y B con la prueba de muestras pareadas de Wilcoxon.

$\uparrow$ Obtenido al comparar columnas B y C con la prueba de Mann-Whitney.

$\mathrm{EI}$ = evaluación inicial; EF = evaluación final; Q1 = primer cuartil 1; Q3 = tercer cuartil.

OSATS = Objective Structured Assessment of Technical Skill; EVOPUC = Escala de Valoración de Otoplastia de la

Pontificia Universidad Católica de Chile; CLA = Cuestionario Likert de Antropometría.

evaluación final y los alcanzados por los expertos se muestran en la Tabla 4 y Figuras 1 a 4 . Los principiantes mejoraron significativamente sus puntajes medios de OSATS y EVOPUC, comparables con los obtenidos por los expertos ( $p<$ 0.05). El tiempo operatorio también se redujo significativamente en la evaluación final de los principiantes ( $p<0.05$ ). Aun así, éstos tardaron más tiempo en completar el procedimiento en comparación con los expertos; no obstante, esta diferencia no fue estadísticamente significativa $(p>0.05)$.

El resultado promedio del CLA también aumentó significativamente después del entrenamiento, obteniendo puntajes similares a los obtenidos por los expertos. La comparación de

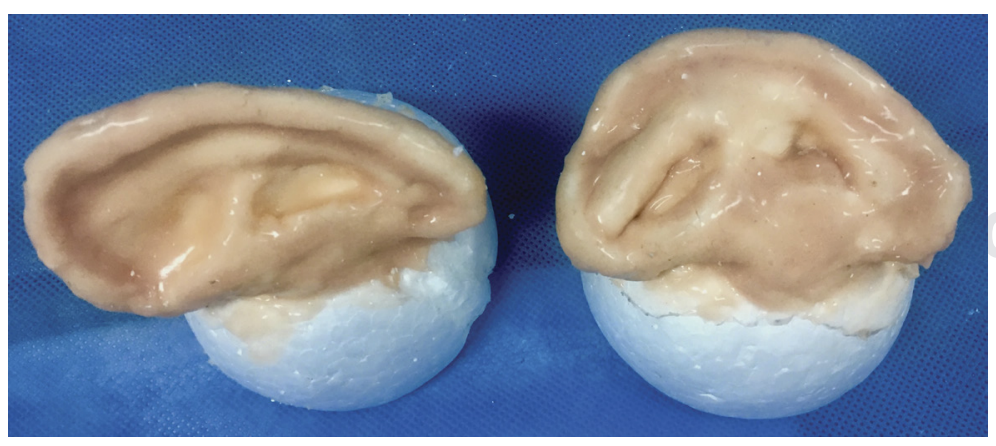

Figura 3: Modelo de otoplastia no operado (izquierda) y resultado postoperatorio realizado por un experto (derecha).
CLA entre los principiantes y expertos después del entrenamiento no fue estadísticamente significativa ( $p>0.05)$. Se puede observar la imagen comparativa entre un modelo no operado y el resultado de uno hecho por un experto en la Figura 3, y la corrección del PAH realizada por un novato antes y después del entrenamiento en la Figura 4. En la Figura 5 se puede comparar la corrección del AAC entre un modelo no operado, uno operado por un experto, uno realizado por un principiante antes y después del entrenamiento.

\section{Validez de contenido y apariencia: encuestas de expertos}

Se hizo una encuesta de ocho preguntas a través de los formularios de Google a los cinco expertos. En términos de realismo del modelo (validez de apariencia), el ítem mejor calificado fue la incisión en la piel y la disección entre los planos de la piel y el cartílago, con una calificación media de 4.8. El elemento peor evaluado fue el plegamiento del antihélix con una puntuación media de 4.0. Con respecto a la validez de contenido, todos los expertos "estuvieron totalmente de acuerdo" en que lo implementarían en la capacitación de sus residentes y lo consideraron útil para la adquisición de habilidades quirúrgicas en otoplastia. Los resultados de la encuesta se pueden revisar en las Tablas 5 y 6 . 

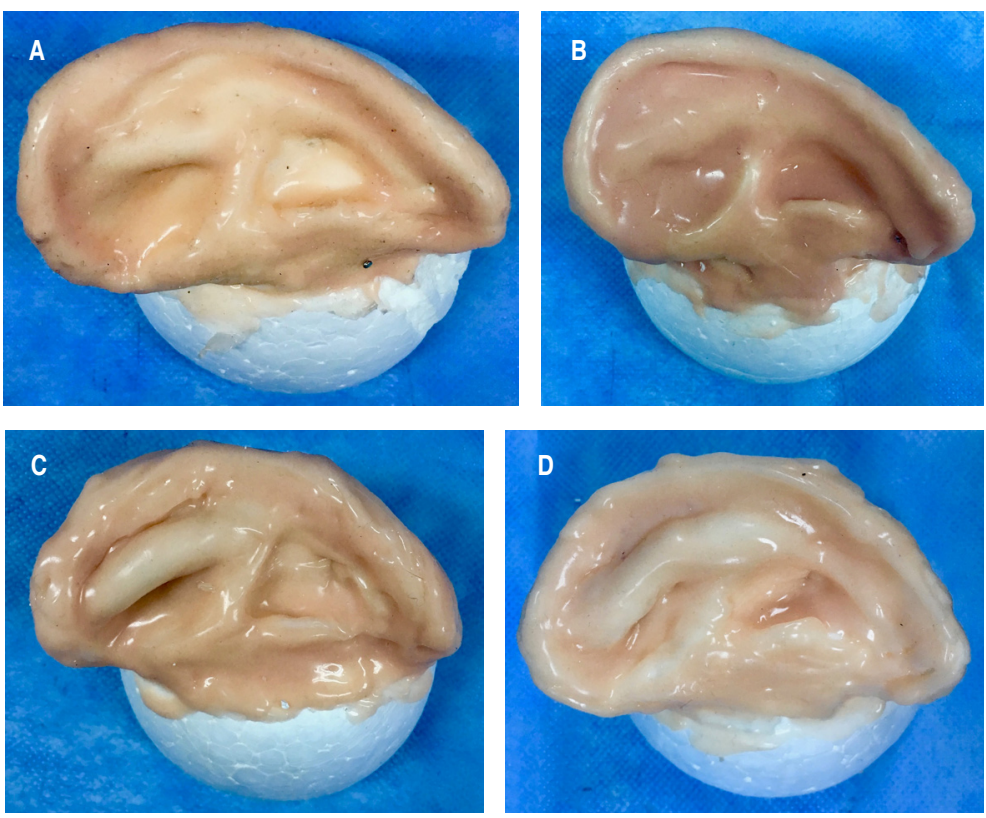

Figuras 4: A y B) Resultados de principiantes previo al entrenamiento. C y D) Resultados de principiantes posterior al entrenamiento.
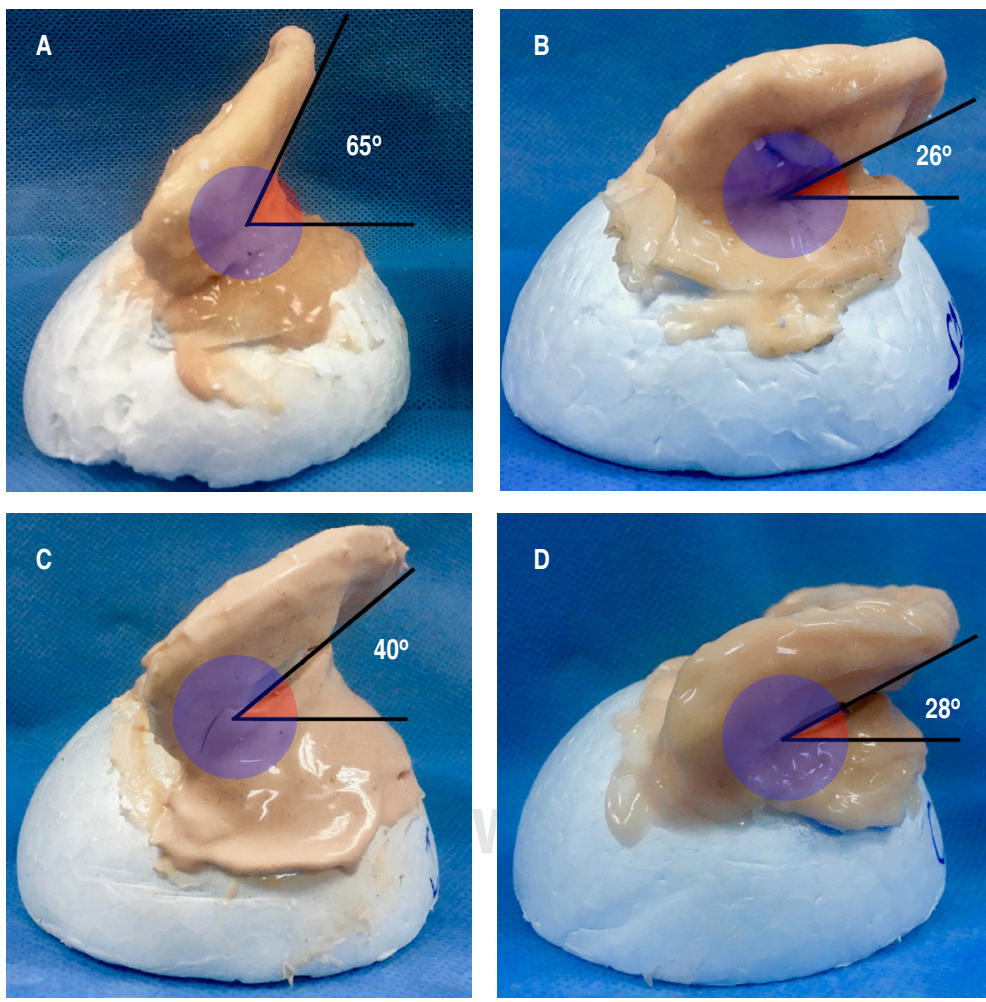

Figura 5: Vista lateral (desde el lóbulo) del modelo de otoplastia que muestra la mejora de $A A C$. A) Modelo no operado, B) Resultado experto, $C$ ) Resultado de principiante previo al entrenamiento, D) Resultado de principiante postentrenamiento.

\section{DISCUSIÓN}

El desarrollo de simuladores en la educación quirúrgica moderna ha cambiado de un modo drástico el modelo clásico de aprendizaje "ver uno, hacer uno, enseñar uno"; este desarrollo ha hecho mayor énfasis en el entrenamiento de simulación preclínica en diversos campos de la cirugía. ${ }^{23,31-33}$ Una de las razones de este cambio es una mayor demanda de seguridad del paciente, lo que ha llevado a menor participación de los residentes en algunos procedimientos quirúrgicos, especialmente en un área como la cirugía estética. Por ello, los esfuerzos por desarrollar y validar de manera adecuada simuladores han sido ampliamente valorados, ya que acortan la curva de entrenamiento y mejoran el rendimiento en el pabellón. ${ }^{23,34-38}$ La dificultad para acceder a modelos cadavéricos humanos o animales de otoplastia, la falta de precisión anatómica y la validación incompleta de los pocos modelos sintéticos publicados hacen de nuestro modelo una propuesta útil y novedosa, además de que su costo de producción es casi 10 veces más barato que el único modelo que, hasta donde sabemos, está disponible en el mercado. ${ }^{18,39}$ Nuestro estudio propone un programa de entrenamiento factible para la adquisición de habilidades quirúrgicas en otoplastia. Aunque las técnicas elegidas y evaluadas fueron las de Furnas y Mustardé, en teoría podrían realizarse otras técnicas de otoplastia y cirugía reconstructiva de pabellón auricular en nuestro modelo de simulación.

Sin embargo, nuestro modelo tiene algunas limitaciones. Una de las críticas de los expertos fue que la piel y el cartílago eran ligeramente más gruesos que en la anatomía real, lo que hace que la definición del antihélix a veces fuese más difícil de conseguir, dado que no están acostumbrados a estas dimensiones. Esto se correlaciona con el hecho de que el plegamiento del antihélix fue el peor calificado en la encuesta, con una media de 4.0 puntos (Tabla 5). Otra limitación es que el proceso de fabricación fue realizado de forma manual, lo que dificulta su reproducibilidad. Además, a pesar de que el valor por modelo fue bajo, no se consideró el tiempo dedicado a diseñar y crear los modelos, ni el tiempo que dedicaron los expertos a las sesiones de retroalimentación, lo que teóricamente aumentaría los costos por el tiempo invertido. Una solución parcial sería optimizar las sesiones a través de videos tutoriales. 
Tabla 5: Encuesta de validez de apariencia. Resultados de expertos.

\begin{tabular}{lcccccc} 
Ítem del cuestionario & $\begin{array}{c}1=\text { muy mal } \\
\mathrm{n}(\%)\end{array}$ & $\begin{array}{c}2=\text { mal } \\
\mathrm{n}(\%)\end{array}$ & $\begin{array}{c}3=\text { promedio } \\
\mathrm{n}(\%)\end{array}$ & $\begin{array}{c}4=\text { bien } \\
\mathrm{n}(\%)\end{array}$ & $\begin{array}{c}5=\text { muy bien } \\
\mathrm{n}(\%)\end{array}$ & Media \\
\hline $\begin{array}{l}\text { Marcación de la piel y } \\
\text { planificación quirúrgica }\end{array}$ & $0(0)$ & $0(0)$ & $0(0)$ & $3(60)$ & $2(40)$ & 4.4 \\
$\begin{array}{l}\text { Incisión en la piel } \\
\begin{array}{l}\text { Disección de la piel del } \\
\text { cartílago y la mastoides }\end{array}\end{array}$ & $0(0)$ & $0(0)$ & $0(0)$ & $1(20)$ & $4(80)$ & 4.8 \\
$\begin{array}{l}\text { Creación del antihélix } \\
\text { (técnica de Mustardé) }\end{array}$ & $0(0)$ & $1(20)$ & $0(0)$ & $2(40)$ & $2(40)$ & 4.6 \\
$\begin{array}{l}\text { Suturas conchomastoida- } \\
\text { les (técnica de Furnas) }\end{array}$ & $0(0)$ & $0(0)$ & $1(20)$ & $2(40)$ & $2(40)$ & 4.2
\end{tabular}

Respecto a la metodología de evaluación del entrenamiento, estamos conscientes de que el tamaño de la muestra fue pequeño. Aun así, obtuvimos resultados positivos y estadísticamente significativos en un grupo homogéneo de principiantes sin conocimientos previos de otoplastia (Video suplementario 2). Los novatos sólo tenían habilidades básicas de sutura, lo que explica por qué el puntaje OSATS fue tan bajo y lo hacía el único aspecto no comparable con el puntaje de los expertos después del entrenamiento, a pesar de que su mejora en el rendimiento fuese significativa. En cuanto a la definición de "expertos", nuestro criterio fue principalmente cualitativo y empírico. Otra variable importante habría sido incluir un grupo intermedio con un grado de experiencia, como los residentes de cirugía plástica. Sin embargo, la razón por la cual los estudiantes de medicina fueron elegidos como el grupo novato fue para asegurar que no existiera experiencia previa a la técnica a ser entrenada, siendo un método común utilizado en la validación de simuladores médicos. ${ }^{20}$

Con respecto a los resultados de la encuesta a expertos, la impresión general del realismo quirúrgico y anatómico fue buena, y todos los expertos coincidieron en que el simulador era una valiosa herramienta de entrenamiento que implementarían para sus residentes.

Este estudio utiliza la mayoría de las estrategias requeridas para validar un programa de simulación como la evaluación inicial de principiantes con instrucción previa, la evaluación inicial y final por evaluadores cegados, el uso de escalas de evaluación generales y específicas, el diseño de un esquema de entrenamiento con retroalimentación efectiva de expertos y un grupo control de expertos.

Tabla 6: Encuesta de validez de contenido. Resultados de expertos.

\begin{tabular}{|c|c|c|c|c|c|c|}
\hline Ítem del cuestionario & $\begin{array}{c}1=\text { muy mal } \\
\mathrm{n}(\%)\end{array}$ & $\begin{array}{c}2=\mathrm{mal} \\
\mathrm{n}(\%)\end{array}$ & $\begin{array}{c}3=\text { promedio } \\
n(\%)\end{array}$ & $\begin{array}{c}4=\text { bien } \\
n(\%)\end{array}$ & $\begin{aligned} 5= & \text { muy bien } \\
& n(\%)\end{aligned}$ & Media \\
\hline $\begin{array}{l}\text { ¿Considera este modelo } \\
\text { útil para aprender } \\
\text { otoplastia? }\end{array}$ & $0(0)$ & $0(0)$ & $0(0)$ & $1(20)$ & $4(80)$ & 4.8 \\
\hline $\begin{array}{l}\text { ¿Cree que este modelo } \\
\text { podría mejorar las } \\
\text { habilidades de otoplastia } \\
\text { en los residentes? }\end{array}$ & $0(0)$ & $0(0)$ & $0(0)$ & $1(20)$ & $4(80)$ & 4.8 \\
\hline $\begin{array}{l}\text { ¿Implementaría este } \\
\text { modelo en el entrena- } \\
\text { miento de sus residentes? }\end{array}$ & $0(0)$ & $0(0)$ & $0(0)$ & $0(0)$ & $5(100)$ & 5 \\
\hline
\end{tabular}




\section{CONCLUSIÓN}

Desarrollamos un nuevo modelo de otoplastia de bajo costo que utilizamos para entrenar un grupo de estudiantes de medicina. La validez de apariencia, constructo y contenido se logró al comparar las puntuaciones de los principiantes antes y después del entrenamiento con las puntuaciones obtenidas por los expertos, demostrando así el realismo quirúrgico, junto con la utilidad del entrenamiento a través de cuestionarios a los expertos. Las habilidades de otoplastia adquiridas por los principiantes entrenados mejoraron significativamente y fueron comparables con las de los cirujanos experimentados.

El modelo de otoplastia presentado podría ser útil para optimizar la curva de aprendizaje de los residentes en su primer acercamiento a escenarios con pacientes reales. Creemos que el desarrollo de futuras investigaciones debería estar dirigido a evaluar la validez de la transferencia de habilidades desde el modelo simulado hasta llegar a pacientes reales.

\section{REFERENCIAS}

1. Naumann A. Otoplasty-techniques, characteristics and risks. GMS Curr Top Otorhinolaryngol Head Neck Surg. 2007; 6: 1-14. Disponible en: http://www. pubmedcentral.nih.gov/articlerender.fcgi?artid=3199 845\&tool = pmcentrez\&rendertype $=$ abstract.

2. Pawar SS, Koch CA, Murakami C. Treatment of prominent ears and otoplasty: a contemporary review. PubMed-NCBI. JAMA Facial Plast Surg. 2015; 17 (6): 449-454. doi: 10.1001/jamafacial.2015.0783.

3. Weerda $\mathrm{H}$. Classification of congenital deformities of the auricle. Facial Plast Surg. 1988; 5: 385-388. doi: 10.1055/s-2008-1064778.

4. Tanzer R. Congenital deformities. In: Converse J, Ed. Reconstructive plastic surgery. Philadelphia: Saunders; 1977 , p. 1671

5. Madzharov MM. A new method of auriculoplasty for protruding ears. Br J Plast Surg. 1989; 42 (3): 285-290. doi: 10.1016/0007-1226(89)90148-3.

6. Wodak E. On the position and shape of the human auricle. Arch Klin Exp Ohren Nasen Kehlkopfheilkd. 1967; 188 (2): 331-335. Disponible en: http://www.ncbi.nlm.nih. gov/pubmed/5599755. Accessed 31 July 2017].

7. Farkas LG. Anthropometry of the normal and defective ear. Clin Plast Surg. 1990; 17 (2): 213-221. http://www. ncbi.nlm.nih.gov/pubmed/2189638.

8. Basat SO, Askeroğlu U, Aksan T, Alleyne B, Yazar M, Orman Ç, et al. New otoplasty approach: a laterally based postauricular dermal flap as an addition to mustarde and furnas to prevent suture extrusion and recurrence. Aesthetic Plast Surg. 2014; 38 (1): 83-89. doi: 10.1007/s00266-013-0269-z.

9. Haytoglu S, Haytoglu TG, Bayar Muluk N, Kuran G, Arikan OK. Comparison of two incisionless otoplasty techniques for prominent ears in children. Int J Pediatr Otorhinolaryngol. 2015; 79 (4): 504-510. doi: 10.1016/j.ijporl.2015.01.014.

10. Mashhadi S, Butler DP. A strategy for assessing otoplasty outcome intra-operatively. J Plast Reconstr Aesthetic Surg. 2012; 65 (7): 984-985. doi: 10.1016/j. bjps.2011.11.056.

11. Shokrollahi K. The discrete scar in prominent ear correction: a digital 3-dimensional analysis to determine the ideal incision for otoplasty: reply. Ann Plast Surg. 2015; 75 (4): 487. doi: 10.1097/ SAP.0000000000000607.

12. Stewart KJ, Lancerotto L. Surgical otoplasty: an evidence-based approach to prominent ears correction. Facial Plast Surg Clin North Am. 2018; 26 (1): 9-18. doi: 10.1016/j.fsc.2017.09.002.

13. Mustarde JC. The correction of prominent ears using simple mattress sutures. Br J Plast Surg. 1963; 16: 170-178. http://www.ncbi.nlm.nih.gov/ pubmed/13936895.

14. Furnas DW. Correction of prominent ears by conchamastoid sutures. Plast Reconstr Surg. 1968; 42 (3): 189-193. http://www.ncbi.nlm.nih.gov/ pubmed/4878456.

15. Loh CYY, Gunn E, Pennell DJL, Athanassopoulos T. Pinnaplasty: a porcine training model. J Plast Reconstr Aesthetic Surg. 2014; 67 (6): 868-869. doi:10.1016/j. bjps.2014.01.012.

16. Uygur S, Ozturk C, Kwiecien G, Siemionow MZ. Sheep head model for plastic surgery training. Plast Reconstr Surg. 2013; 132 (5): 895e-896e. doi:10.1097/ COC.0b013e318210f83c.

17. L Wu, K Pang, Y Ng, S Yeak. Proposed otoplasty model: innovative use for a hearing aid demonstration mould. The Internet Journal of Plastic Surgery. 2006; 3 (1): 1-4.

18. Schneider G, Voigt S, Rettinger G. Computed tomography-based training model for otoplasty. Eur Arch Oto-Rhino-Laryngology. 2016; 273 (9): 24272432. doi: 10.1007/s00405-015-3797-0.

19. Guieiro M, Guimarães R, Ricardo L, Souto M. Pinna synthetic mold for otoplasty techniques application. Braz J Otorhinolaryngol. 2018; 84 (2): 159-165. doi: 10.1016/j.bjorl.2017.01.004.

20. McDougall EM. Validation of surgical simulators. J Endourol. 2007; 21 (3): 244-247. doi: 10.1089/ end.2007.9985.

21. Singh $M$, Ziolkowski N, Ramachandran S, Myers SR, Ghanem AM. Development of a five-day basic microsurgery simulation training course: A cost analysis. Arch Plast Surg. 2014; 41 (3): 213-217. doi: 10.5999/aps.2014.41.3.213.

22. Ramos P, Montez J, Tripp A, Ng CK, Gill IS, Hung AJ. Face, content, construct and concurrent validity of dry laboratory exercises for robotic training using a global assessment tool. BJU Int. 2014; 113 (5): 836-842. doi: 10.1111/bju.12559.

23. Zevin B, Levy JS, Satava RM, Grantcharov TP. A consensus-based framework for design, validation, and implementation of simulation-based training curricula in surgery. J Am Coll Surg. 2012; 215 (4): 580-586. doi: 10.1016/j.jamcollsurg.2012.05.035.

24. Carey JN, Rommer E, Sheckter C, Minneti M, Talving $\mathrm{P}$, Wong AK, et al. Simulation of plastic surgery and 
microvascular procedures using perfused fresh human cadavers. J Plast Reconstr Aesthetic Surg. 2014; 67 (2): e42-e48. doi: 10.1016/j.bjps.2013.09.026

25. Mason KF. Changes in the wind. Compos Technol. 2004; 10 (2): 26-31. doi: 10.1021/jf052913o.

26. Rodriguez JR, Yañez R, Cifuentes I, Varas J, Dagnino B. Microsurgery workout : a novel simulation. Plast Reconstr Surg. 2016;.138: 739-747. doi: 10.1097/ PRS.0000000000002456.

27. Martin JA, Regehr G, Reznick R, MacRae H, Murnaghan J, Hutchison C, et al. Objective structured assessment of technical skill (OSATS) for surgical residents. Br J Surg. 1997; 84 (2): 273-278. doi: 10.1002/bjs.1800840237.

28. Urrutia J, Besa P, Campos M, Cikutovic P, Cabezon M, Molina M, et al. The Pfirrmann classification of lumbar intervertebral disc degeneration: an independent interand intra-observer agreement assessment. Eur Spine J. 2016; 25 (9): 2728-2733. doi: 10.1007/s00586-0164438-z.

29. Fleiss J. The design and analysis of clinical experiments. New York: Wiley; 1986.

30. Cicchetti D V. Guidelines, criteria, and rules of thumb for evaluating normed and standardized assessment instruments in psychology. Psychol Assess. 1994; 6 (4): 284-290. doi: 10.1037/1040-3590.6.4.284.

31. Sinceri S, Berchiolli R, Marconi M, Cioni R, Ferrari V, Ferrari $\mathrm{M}$, et al. Face, content, and construct validity of a simulator for training in endovascular procedures. Minim Invasive Ther Allied Technol. 2018; 27 (6): 315-320. doi: 10.1080/13645706.2018.1458038.

32. Olabe J, Olabe J. Microsurgical training on an in vitro chicken wing infusion model. Surg Neurol. 2009; 72 (6): 695-699. doi: 10.1016/j.surneu.2008.12.008.

33. Varas J, Mejía R, Riquelme A, Maluenda F, Buckel E, Salinas J, et al. Significant transfer of surgical skills obtained with an advanced laparoscopic training program to a laparoscopic jejunojejunostomy in a live porcine model: feasibility of learning advanced laparoscopy in a general surgery residency. Surg Endosc. 2012; 26 (12): 3486-3494. doi: 10.1007/ s00464-012-2391-4.
34. Demirseren ME, Ceran C, Duman Y, Sarici M. Excised abdominoplasty material as a systematic plastic surgical training model. Plast Surg Int. 2012;2012:834212. doi: 10.1155/2012/834212.

35. Zucca-Matthes G, Lebovic G, Lyra M. Mastotrainer new version: realistic simulator for training in breast surgery. Breast. 2017; 31: 82-84. doi: 10.1016/j. breast.2016.08.009.

36. Denadai R, Toledo AP, Martinhão Souto LR. Basic plastic surgery skills training program on inanimate bench models during medical graduation. Plast Surg Int. 2012; 2012: 1-12. doi: 10.1155/2012/651863.

37. Al-Bustani S, Halvorson EG. Status of microsurgical simulation training in plastic surgery: a survey of United States Program Directors. Ann Plast Surg. 2016; 76 (6): 713-716. doi: 10.1097/SAP.0000000000000636.

38. Rosen JM, Long SA, McGrath DM GS. Simulation in plastic surgery training and education: the path forward. Plast Reconstr Surg. 2009; 123 (2): 739-740. doi: 10.1097/PRS.0b013e3181958f27.

39. Reis MGA dos, Marim RG, Souto LRM. Pinna synthetic mold for otoplasty techniques application. Braz J Otorhinolaryngol. 2018; 84 (2): 159-165. doi: 10.1016/j.bjorl.2017.01.004.

Financiamiento: Este trabajo fue financiado por Fondos de la Dirección de Investigación y Doctorado de la Facultad de Medicina de la Pontificia Universidad Católica de Chile (Núm. PB 23/18) obtenido por el autor Dr. Alfonso Navia.

\section{Correspondencia: \\ Dr. Alfonso Navia Torelli}

Diagonal Paraguay 362, 8330077,

Santiago de Chile, Chile.

Teléfono: +56982094566

E-mail: ajnavia@uc.cl 